\title{
SOME ANNUITY PROBLEMS IN THE FRAMEWORK OF CZECH PENSION SYSTEMS
}

\author{
Tomáš Cipra*
}

\begin{abstract}
:
The contribution analyzes some life annuity aspects of the pension system (including commercial insurance) in the Czech Republic. In particular, the problem of sustainability, the Generation Life Tables for the pension system and the gender problem for the pensions in the Czech Republic are discussed. Such topics are important for the future of the pension system in the Czech Republic and for the necessary pension reform. Some numerical results with the Czech data concerning these aspects are presented including corresponding conclusions. Economic theory of pensions (see e.g. Sheshinski, 2005 or Uebelmesser, 2004) is not considered here.
\end{abstract}

Keywords: Generation Life Tables, life annuities, longevity risk, pension system, sustainability of pensions, Unisex Life Tables

JEL Classification: D14, D30, G22, H55

\section{Introduction}

One of the serious risks for the future society is the so called longevity, i.e. a social insufficiency due to as a consequence of the old age. The concept of longevity risk is the key topic for the pension reform planned in the Czech Republic and delayed till the time moment when the demographic situation becomes critical (see e.g. the comparison of the age distribution of the Czech population in 1950 and 2008 in Figure 1). The numbers of survivors till particular ages from the Life Tables of the Czech Republic in 2010 are given in Figure 2.

* Faculty of Mathematics and Physics, Charles University of Prague, Sokolovská 83, CZ - 18675 Prague 8 (cipra@karlin.mff.cuni.cz).

This paper has been written with the support of the Czech Science Foundation No. P402/12/G097 "DYME - Dynamic Models in Economics". 
Figure 1

Comparison of the Age Distribution of the Czech Population in 1950 and 2008 Age Distribution of the Population: 1950

Czech Republic

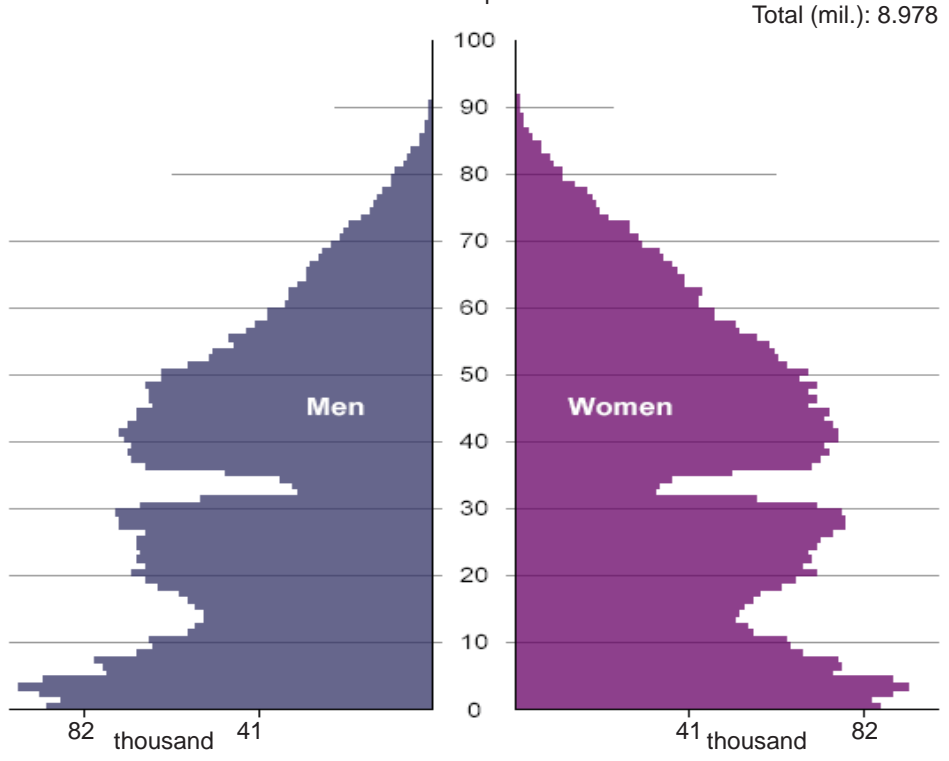

Age Distribution of the Population: 2008

Czech Republic

Total (mil.): 10.468
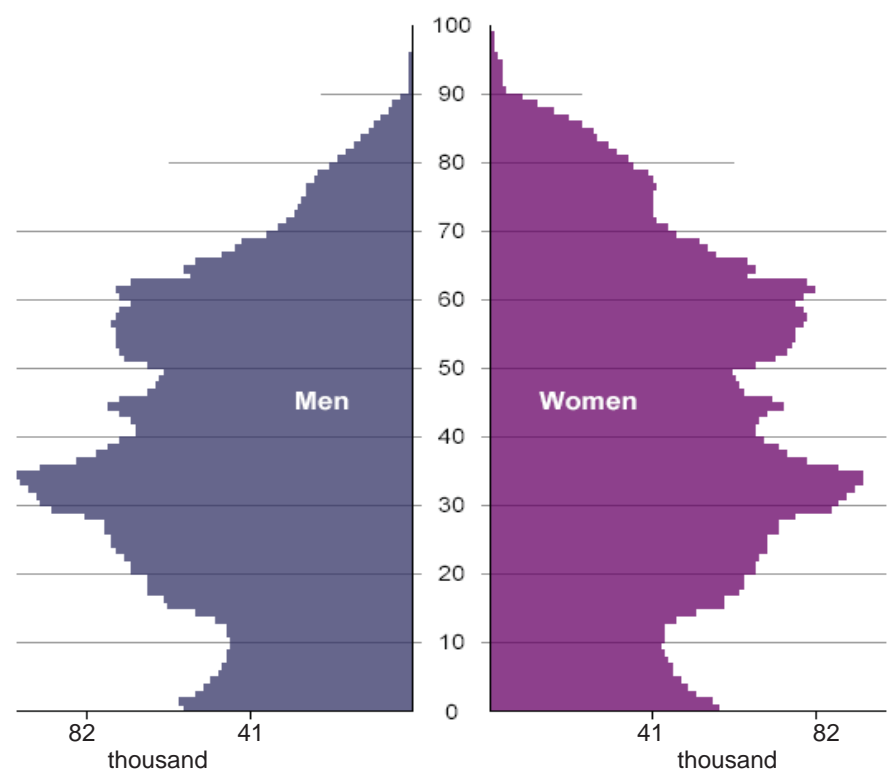

Source: Eurostat, 2008. 
One must stress in this framework that the pension assets (i.e. capitalized claims for all types of pensions of a given individual which can be further classified to public, occupational and private pensions) become important part of the personal assets wealth in the modern society. Besides the qualitative aspects (e.g. the proportion of pension assets in the volume of the personal wealth, see Blake and Orszag, 1999; Cannon and Tonks, 2008; Clark, Munnell and Orszag, 2006 and others) there are quantitative questions that should be solved in this context:

(1) The sustainability of pensions: One investigates (under the given tolerance and with the given investment efficiency) which regular annuity spending (spending rate) is admissible for a given pension account. For this purpose special pension models may be used which are presented here and which are applied numerically to pension systems in the Czech Republic to provide important conclusions (see Section 2).

(2) Generation Life Tables (GLT): In the pension context one should apply special Life Tables with projections distinguishing among particular generations (cohorts). Moreover, these tables should be adjusted in a special way to be suitable for pensions (see Section 3 ).

(3) Unisex Life Tables (ULT): Due to various anti-discrimination laws concerning the insurance the ULT should be constructed for the Czech population (see Section 4).

Figure 2

Numbers of Survivors till Particular Ages from the Life Tables of the Czech Republic in 2010

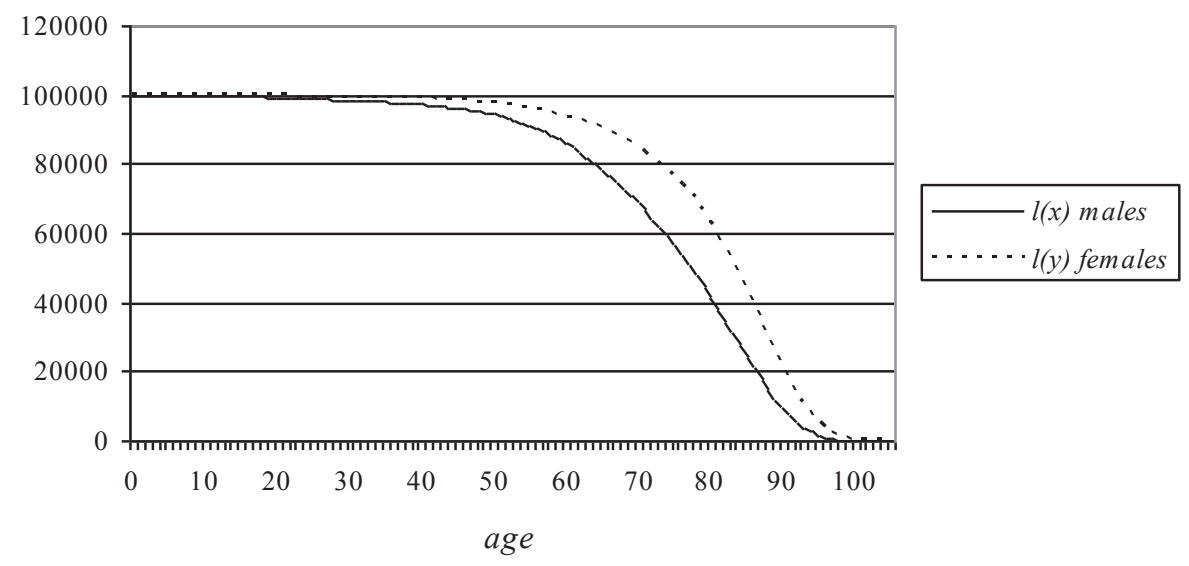

Source: Czech Statistical Office, 2010.

\section{Sustainability of Pensions}

If a pension system is based on the fund principle then a question appears how much to "save" annually during the accumulation phase and how much to "spend" annually during the decumulation (annuity) phase. In view of many random aspects the best approach is similar to the one applied in modern finance, namely the Value-at-Risk (VaR, 
see e.g. Cipra, 2010), when one calculates the highest loss which can occur with a given probability (tolerance). In the pension context it must be modified to the probability that the retired person will not be "ruined" before the moment of death (such a probability can be called the probability of sustainable pension) while its complement to 1 is the probability of ruin (or the probability of unsustainable pension). In the practice it is closely connected with the pension planning (pension consultants speak sometimes on pension risk management).

For simplicity let us consider only the so called DC pension plans (Defined Contribution) where the contributions to the system are defined in advance (frequently by a percentage of the participant's salary). In the age of retirement (e.g. in 65) there is an accumulated capital $w$ on the participant's account which will be decumulated by corresponding annual pension payments. In particular, the investment risk is fully on the side of the participants of the pension plan (not on the side of the pension provider). Two aspects of random character should be at least considered:

(1) Randomness of interest rates for investment of the capital from the participant's account is in the modern finance usually modelled by means of the geometric Brown motion (i.e. exponential Wiener process, see e.g. Cipra, 2008 or Malliaris and Brock, 1982). Here the capital $S_{t}$ in time $t$ can be evaluated starting with the capital $S_{0}$ (in time 0 ) as

$$
S_{t}=S_{0} \cdot e^{W_{t}^{(\mu, \sigma)}}=S_{0} \cdot e^{\mu \cdot t+\sigma \cdot W_{t}},
$$

where $W_{t}$ is the classical Wiener process, $\mu$ is the drift modelling the trend of the capital investment and $\sigma$ is the volatility modelling the diffusion of the capital investment. In particular, $S_{t}$ is log-normally distributed

$$
\ln S_{t} \sim N\left(\ln S_{0}+\mu \cdot t, \sigma^{2} t\right)
$$

with the mean value

$$
E\left(S_{t}\right)=S_{0} \cdot e^{\left(\mu+\frac{\sigma^{2}}{2}\right) \cdot t}=S_{0} \cdot e^{\nu \cdot t}
$$

and the median value

$$
M\left(S_{t}\right)=S_{0} \cdot e^{\mu \cdot t}
$$

(2) Randomness of the future lifetime $T_{x}$ of an individual aged $x$ can be modelled in the simplest case by the exponential law of mortality:

$$
{ }_{t} p_{x}=\exp \left\{-\int_{x}^{x+t} \lambda_{x} d s\right\}=\exp \left\{-\lambda_{x} \cdot t\right\},
$$

where $\lambda_{x}$ is the force of mortality at age $x$ (i.e. an infinitesimal version of the probability of death at the given age). In particular, the expected remaining lifetime (the life expectancy) at age $x$ is 


$$
e_{x}=E\left(T_{x}\right)=\frac{1}{\lambda_{x}}
$$

and the median remaining lifetime at age $x$ is

$$
M\left(T_{x}\right)=\frac{\ln (2)}{\lambda_{x}}
$$

(see e.g. Milevsky, 2006). If we combine the models (1) and (5) we'll obtain the present value $P V_{x}$ of the standard pension (which pay unit annual payments in continuous time) as random variable

$$
P V_{x}=\int_{0}^{T_{x}} e^{-\left(\mu \cdot t+\sigma \cdot W_{t}\right)} d t
$$

Finally one can show (see e.g. Dufresne, 1990; Milevsky, 1997; Milevsky, 2006 that the probability of ruin (the probability of unsustainable pension)

$$
P\left(P V_{x}>w\right)=P\left(\int_{0}^{T_{x}} e^{-\left(\mu \cdot t+\sigma \cdot W_{t}\right)} d t>w\right)
$$

( $w>0$ is the sum on the participant's account at the age of retirement $x$ ) can be approximated as

$$
P\left(P V_{x}>w\right) \sim \frac{1}{\beta^{\alpha} \Gamma(\alpha)} \int_{0}^{1 / w} z^{\alpha-1} \exp \left(-\frac{z}{\beta}\right) d z=1-\frac{1}{\beta^{\alpha} \Gamma(\alpha)} \int_{0}^{w} y^{-(\alpha+1)} \exp \left(-\frac{1}{\beta y}\right) d y,(10)
$$

where

$$
\alpha=\frac{2 \mu+4 \lambda_{x}}{\sigma^{2}+\lambda_{x}}-1, \beta=\frac{\sigma^{2}+\lambda_{x}}{2}
$$

and $\Gamma(\alpha)$ is the gamma function

$$
\Gamma(\alpha)=\int_{0}^{\infty} z^{\alpha-1} e^{-z} d z .
$$

Moreover, the mean present value $\mathrm{E}\left(P V_{x}\right)$ can be approximated as

$$
E\left(P V_{x}\right) \sim \frac{1}{\mu-\sigma^{2}+\lambda_{x}} .
$$

The previous formulas enable to perform the corresponding calculations for pension plans in the Czech Republic. For this purpose one must use both financial and longevity (or equivalently mortality) data:

(1) Financial data: For the purpose of the investment formula (1) one could use the so called technical interest rate $2.5 \%$ used for insurance calculations according to 
the insurance legislative in the Czech Republic in year 2012 (correctly speaking, it is the upper limit in the case of long-term insurance products in the commercial life insurance including life annuities holding since 2010). The better strategy is to test more scenarios for the drift $\mu$ using the technical interest rate $2.5 \%$ as one of possibilities (see e.g. Table 3 or Figures 3 and 4).

(2) Longevity data: We have used Life Tables (LT) for males and females in the Czech Republic in 2010. From the expected remaining lifetime (the life expectancy) $e_{x}$ at particular ages $x$ given in these LT one estimates according to the formula (6) very simply the parameters $\lambda_{x}$ (see Table 1). One could obtain better estimates of longevity applying Gompertz-Makeham law of mortality or even Lee-Carter approach (instead of our exponential law of mortality) but the exponential approach seems to be for our calculations sufficient.

Table 1

The Instantaneous Forces of Mortality of the Exponential Law of Mortality for Males and Females in the Czech Republic in 2010

\begin{tabular}{|l|c|c|c|c|}
\hline & \multicolumn{2}{|c|}{ Males CZ 2010 } & \multicolumn{2}{c|}{ Females CZ 2010 } \\
\hline $\boldsymbol{x}$ and $\boldsymbol{y}$ & $\boldsymbol{e}_{\boldsymbol{x}}$ & $\boldsymbol{\lambda}_{\boldsymbol{x}}$ & $\boldsymbol{e}_{\boldsymbol{y}}$ & $\boldsymbol{\lambda}_{\boldsymbol{y}}$ \\
\hline $\mathbf{5 5}$ & 22.50 & 0.0444 & 27.31 & 0.0366 \\
\hline $\mathbf{6 0}$ & 18.69 & 0.0535 & 22.91 & 0.0436 \\
\hline $\mathbf{6 5}$ & 15.26 & 0.0655 & 18.71 & 0.0534 \\
\hline $\mathbf{7 0}$ & 12.11 & 0.0826 & 14.77 & 0.0677 \\
\hline $\mathbf{7 5}$ & 9.16 & 0.1092 & 11.12 & 0.0899 \\
\hline $\mathbf{8 0}$ & 6.62 & 0.1511 & 7.91 & 0.1264 \\
\hline $\mathbf{8 5}$ & 4.54 & 0.2203 & 5.32 & 0.1880 \\
\hline
\end{tabular}

In Tables 2-4 we have calculated according to (9) the probability of ruin (i.e. the probability of unsustainable pension) for various retirement ages $55,60, \ldots, 85$ in dependence on the spending rates $1 / w$ (e.g. the spending rate 0.06 means that the given pension account of CZK 1,000,000 (Czech korunas) will pay CZK60,000 annually, i.e. CZK 5,000 monthly); the calculations are performed separately for males and females and for various values of investment drifts and volatilities, namely $\mu=1 \%$ and $\sigma=5 \%$ in Table $2, \mu=2.5 \%$ and $\sigma=5 \%$ in Table $3, \mu=5 \%$ and $\sigma=10 \%$ in Table 4 (the results of these tables are interpreted from the practical point of view in the end of this section): 
Table 2

The Probability of Ruin (i.e. the probability of unsustainable pension in per cent) for Various Retirement Ages and Spending Rates: for $\mu=1 \%$ and $\sigma=5 \%$

\begin{tabular}{|c|c|c|c|c|c|c|c|c|c|c|c|c|}
\hline \multicolumn{13}{|c|}{ Males CZ 2010 for $\mu=1 \%, \sigma=5 \%$ : probability of unsustainable pension (\%) } \\
\hline \multirow[b]{2}{*}{$x$} & \multirow[b]{2}{*}{$e_{x}$} & \multirow[b]{2}{*}{$\lambda$} & \multicolumn{10}{|c|}{ Spending rate $1 / \mathrm{w}$ : } \\
\hline & & & 0.01 & 0.02 & 0.03 & 0.04 & 0.05 & 0.06 & 0.07 & 0.08 & 0.09 & 0.10 \\
\hline 55 & 22.50 & 0.0444 & $0.6 \%$ & $4.0 \%$ & $10.8 \%$ & $20.2 \%$ & $31.0 \%$ & $41.9 \%$ & $52.2 \%$ & $61.5 \%$ & $69.5 \%$ & $76.2 \%$ \\
\hline 60 & 18.69 & 0.0 & $0.4 \%$ & 2 & $\%$ & $14.4 \%$ & $22.9 \%$ & $32.1 \%$ & $41.3 \%$ & $50.1 \%$ & $58.2 \%$ & $65.5 \%$ \\
\hline 65 & 15.26 & 0.0655 & $0.2 \%$ & $1.7 \%$ & $4.8 \%$ & $9.7 \%$ & $15.9 \%$ & $23.0 \%$ & $30.5 \%$ & $38.2 \%$ & $45.7 \%$ & $52.8 \%$ \\
\hline 70 & 12.11 & 0.0826 & $0.1 \%$ & $1.0 \%$ & $2.9 \%$ & $5.9 \%$ & $10.0 \%$ & $15.0 \%$ & $20.6 \%$ & $26.6 \%$ & $32.7 \%$ & $38.9 \%$ \\
\hline 75 & 9.16 & 0.1092 & $0.1 \%$ & $0.5 \%$ & $1.5 \%$ & $3.1 \%$ & $5.5 \%$ & $8.4 \%$ & $12.0 \%$ & $15.9 \%$ & $20.3 \%$ & $24.8 \%$ \\
\hline 80 & 6.62 & 0.1511 & $0.0 \%$ & $0.2 \%$ & $0.6 \%$ & $1.4 \%$ & $2.5 \%$ & $4.0 \%$ & $5.9 \%$ & $8.1 \%$ & $10.6 \%$ & $13.4 \%$ \\
\hline 85 & 4.54 & 0.2203 & $0.0 \%$ & $0.1 \%$ & $0.2 \%$ & $0.5 \%$ & $1.0 \%$ & $1.6 \%$ & $2.4 \%$ & $3.4 \%$ & $4.5 \%$ & $5.9 \%$ \\
\hline
\end{tabular}

\begin{tabular}{|c|c|c|c|c|c|c|c|c|c|c|c|c|}
\hline \multicolumn{13}{|c|}{ Females CZ 2010 for $\mu=1 \%, \sigma=5 \%$ : probability of unsustainable pension (\%) } \\
\hline \multirow[b]{2}{*}{$y$} & \multirow[b]{2}{*}{$\mathbf{e}_{y}$} & \multirow[b]{2}{*}{$\boldsymbol{\lambda}$} & \multicolumn{10}{|c|}{ Spending rate $1 / \mathrm{w}$ : } \\
\hline & & & 0.01 & 0.02 & 0.03 & 0.04 & 0.05 & 0.06 & 0.07 & 0.08 & 0.09 & 0.10 \\
\hline 55 & 27.31 & 0.0366 & $0.9 \%$ & $6.0 \%$ & $15.6 \%$ & $27.9 \%$ & $40.9 \%$ & $53.2 \%$ & $64.0 \%$ & $72.9 \%$ & $80.0 \%$ & $85.5 \%$ \\
\hline 60 & 22.91 & 0.0436 & $0.6 \%$ & $4.2 \%$ & $11.2 \%$ & $20.9 \%$ & $31.8 \%$ & $42.9 \%$ & $53.3 \%$ & $62.6 \%$ & $70.6 \%$ & $77.2 \%$ \\
\hline 65 & 18.71 & 0.0534 & $0.4 \%$ & $2.7 \%$ & $7.5 \%$ & $14.5 \%$ & $22.9 \%$ & $32.1 \%$ & $41.3 \%$ & $50.2 \%$ & $58.3 \%$ & $65.5 \%$ \\
\hline 70 & 14.77 & 0.0677 & $0.2 \%$ & $1.5 \%$ & $4.5 \%$ & $9.0 \%$ & $14.9 \%$ & $21.7 \%$ & $29.0 \%$ & $36.4 \%$ & $43.8 \%$ & $50.8 \%$ \\
\hline 75 & 11.12 & 0.0899 & $0.1 \%$ & $0.8 \%$ & $2.3 \%$ & $4.9 \%$ & $8.4 \%$ & $12.6 \%$ & $17.6 \%$ & $22.9 \%$ & $28.5 \%$ & $34.2 \%$ \\
\hline 80 & 7.91 & 0.1264 & $0.0 \%$ & $0.3 \%$ & $1.0 \%$ & $2.2 \%$ & $3.9 \%$ & $6.1 \%$ & $8.8 \%$ & $11.9 \%$ & $15.3 \%$ & $19.0 \%$ \\
\hline 85 & 5.32 & 0.1880 & $0.0 \%$ & $0.1 \%$ & $0.4 \%$ & $0.8 \%$ & $1.5 \%$ & $2.4 \%$ & $3.5 \%$ & $4.9 \%$ & $6.6 \%$ & $8.4 \%$ \\
\hline
\end{tabular}

Table 3

The Probability of Ruin (i.e. the probability of unsustainable pension in per cent) for Various Retirement Ages and Spending Rates: for $\mu=2.5 \%$ and $\sigma=5 \%$

\begin{tabular}{|c|c|c|c|c|c|c|c|c|c|c|c|c|}
\hline \multicolumn{7}{|c|}{ Males CZ 2010 for $\boldsymbol{\mu}=\mathbf{2 . 5} \%, \boldsymbol{\sigma}=\mathbf{5} \%$ : probability of unsustainable pension (\%) } \\
\hline & & & \multicolumn{8}{|c|}{ Spending rate 1/ $\mathbf{~ : ~}$} \\
\cline { 5 - 14 } $\boldsymbol{x}$ & $\mathbf{e}_{\boldsymbol{x}}$ & $\boldsymbol{1}$ & $\mathbf{0 . 0 1}$ & $\mathbf{0 . 0 2}$ & $\mathbf{0 . 0 3}$ & $\mathbf{0 . 0 4}$ & $\mathbf{0 . 0 5}$ & $\mathbf{0 . 0 6}$ & $\mathbf{0 . 0 7}$ & $\mathbf{0 . 0 8}$ & $\mathbf{0 . 0 9}$ & $\mathbf{0 . 1 0}$ \\
\hline $\mathbf{5 5}$ & 22.50 & 0.0444 & $0.1 \%$ & $1.4 \%$ & $5.0 \%$ & $10.9 \%$ & $18.9 \%$ & $28.2 \%$ & $37.9 \%$ & $47.5 \%$ & $56.4 \%$ & $64.4 \%$ \\
\hline $\mathbf{6 0}$ & 18.69 & 0.0535 & $0.1 \%$ & $1.0 \%$ & $3.6 \%$ & $8.0 \%$ & $14.1 \%$ & $21.5 \%$ & $29.6 \%$ & $37.9 \%$ & $46.2 \%$ & $54.0 \%$ \\
\hline $\mathbf{6 5}$ & 15.26 & 0.0655 & $0.1 \%$ & $0.7 \%$ & $2.5 \%$ & $5.5 \%$ & $9.9 \%$ & $15.4 \%$ & $21.8 \%$ & $28.6 \%$ & $35.6 \%$ & $42.6 \%$ \\
\hline $\mathbf{7 0}$ & 12.11 & 0.0826 & $0.0 \%$ & $0.5 \%$ & $1.6 \%$ & $3.5 \%$ & $6.5 \%$ & $10.2 \%$ & $14.7 \%$ & $19.8 \%$ & $25.3 \%$ & $31.0 \%$ \\
\hline $\mathbf{7 5}$ & 9.16 & 0.1092 & $0.0 \%$ & $0.3 \%$ & $0.9 \%$ & $2.0 \%$ & $3.7 \%$ & $5.9 \%$ & $8.7 \%$ & $12.0 \%$ & $15.7 \%$ & $19.7 \%$ \\
\hline $\mathbf{8 0}$ & 6.62 & 0.1511 & $0.0 \%$ & $0.1 \%$ & $0.4 \%$ & $1.0 \%$ & $1.8 \%$ & $3.0 \%$ & $4.4 \%$ & $6.3 \%$ & $8.4 \%$ & $10.7 \%$ \\
\hline $\mathbf{8 5}$ & 4.54 & 0.2203 & $0.0 \%$ & $0.0 \%$ & $0.2 \%$ & $0.4 \%$ & $0.7 \%$ & $1.2 \%$ & $1.9 \%$ & $2.7 \%$ & $3.7 \%$ & $4.8 \%$ \\
\hline
\end{tabular}


Table 3

\begin{tabular}{|c|c|c|c|c|c|c|c|c|c|c|c|c|}
\hline \multicolumn{13}{|c|}{ Females CZ 2010 for $\mu=2.5 \%, \sigma=5 \%$ : probability of unsustainable pension (\%) } \\
\hline \multirow[b]{2}{*}{$y$} & \multirow[b]{2}{*}{$e_{y}$} & \multirow[b]{2}{*}{$\lambda$} & \multicolumn{10}{|c|}{ Spending rate $1 / \mathrm{w}$ : } \\
\hline & & & 0.01 & 0.02 & 0.03 & 0.04 & 0.05 & 0.06 & 0.07 & 0.08 & 0.09 & 0.10 \\
\hline 55 & 27.31 & 0.0366 & $0.2 \%$ & $2.0 \%$ & $6.8 \%$ & $14.8 \%$ & $25.1 \%$ & $36.3 \%$ & $47.6 \%$ & $57.9 \%$ & $67.0 \%$ & $74.7 \%$ \\
\hline 60 & 22.91 & 0.0436 & $0.1 \%$ & $1.5 \%$ & $5.1 \%$ & $11.3 \%$ & $19.5 \%$ & $28.9 \%$ & $38.8 \%$ & $48.5 \%$ & $57.4 \%$ & $65.4 \%$ \\
\hline 65 & 18.71 & 0.0534 & $0.1 \%$ & $1.0 \%$ & $3.6 \%$ & $8.0 \%$ & $14.1 \%$ & $21.5 \%$ & $29.6 \%$ & $38.0 \%$ & $46.2 \%$ & $54.0 \%$ \\
\hline 70 & 14.77 & 0.0677 & $0.1 \%$ & $0.7 \%$ & $2.3 \%$ & $5.2 \%$ & $9.4 \%$ & $14.6 \%$ & $20.7 \%$ & $27.2 \%$ & $34.1 \%$ & $40.9 \%$ \\
\hline 75 & 11.12 & 0.0899 & $0.0 \%$ & $0.4 \%$ & $1.3 \%$ & $3.0 \%$ & $5.5 \%$ & $8.7 \%$ & $12.6 \%$ & $17.1 \%$ & $22.0 \%$ & $27.2 \%$ \\
\hline 80 & 7.91 & 0.1264 & $0.0 \%$ & $0.2 \%$ & $0.6 \%$ & $1.4 \%$ & $2.7 \%$ & $4.4 \%$ & $6.5 \%$ & $9.0 \%$ & $11.9 \%$ & $15.1 \%$ \\
\hline 85 & 5.32 & 0.1880 & $0.0 \%$ & $0.1 \%$ & $0.2 \%$ & $0.6 \%$ & $1.1 \%$ & $1.8 \%$ & $2.7 \%$ & $3.9 \%$ & $5.2 \%$ & $6.8 \%$ \\
\hline
\end{tabular}

Table 4

The Probability of Ruin (i.e. the probability of unsustainable pension in per cent) for Various Retirement Ages and Spending Rates: for $\mu=5 \%$ and $\sigma=10 \%$

\begin{tabular}{|c|c|c|c|c|c|c|c|c|c|c|c|c|}
\hline \multicolumn{13}{|c|}{ Males CZ 2010 for $\mu=5 \%, \sigma=10 \%$ : probability of unsustainable pension (\%) } \\
\hline \multirow[b]{2}{*}{$x$} & & & \multicolumn{10}{|c|}{ Spending rate $1 / \mathrm{w}$ : } \\
\hline & & & 0.01 & 0.02 & 0.03 & 0.04 & 0.05 & 0.06 & 0.07 & 0.08 & 0.09 & 0.10 \\
\hline 55 & 22.50 & 0.0444 & $0.0 \%$ & 0.6 & $2.2 \%$ & $5.5 \%$ & $10.4 \%$ & $16.7 \%$ & $24.0 \%$ & $31.9 \%$ & $40.0 \%$ & $47.9 \%$ \\
\hline 60 & 18.69 & 0.0535 & $0.0 \%$ & $0.4 \%$ & $1.7 \%$ & $4.2 \%$ & $8.0 \%$ & $13.0 \%$ & $19.0 \%$ & $25.6 \%$ & $32.7 \%$ & $39.8 \%$ \\
\hline 65 & 15.26 & 0.0655 & $0.0 \%$ & 0.3 & $1.3 \%$ & $3.1 \%$ & $\%$ & $9.7 \%$ & $14.3 \%$ & $19.6 \%$ & $25.4 \%$ & $31.4 \%$ \\
\hline 70 & 12.11 & 0.06 & $0.0 \%$ & $0.2 \%$ & $0.9 \%$ & 2.1 & $\%$ & $6.7 \%$ & $10.0 \%$ & $13.9 \%$ & $18.3 \%$ & $23.0 \%$ \\
\hline 75 & 9.16 & 0.1092 & $0.0 \%$ & $0.1 \%$ & $0.5 \%$ & $1.3 \%$ & $2.4 \%$ & 4.110 & 0.2 & $8.7 \%$ & $11.7 \%$ & $14.9 \%$ \\
\hline 80 & 6.62 & 0.1511 & $0.0 \%$ & $0.1 \%$ & $0.3 \%$ & $0.7 \%$ & $1.3 \%$ & $2.2 \%$ & $3.3 \%$ & $4.7 \%$ & $6.4 \%$ & $8.4 \%$ \\
\hline 85 & 4.54 & 0.2203 & $0.0 \%$ & $0.0 \%$ & $0.1 \%$ & $0.3 \%$ & $0.6 \%$ & $1.0 \%$ & $1.5 \%$ & $2.2 \%$ & $3.0 \%$ & $3.9 \%$ \\
\hline
\end{tabular}

\begin{tabular}{|c|c|c|c|c|c|c|c|c|c|c|c|c|}
\hline \multicolumn{13}{|c|}{ emales CZ 2010 for $\mu=5 \%, \sigma=10 \%$ : probability of unsustainable pension (\%) } \\
\hline \multirow[b]{2}{*}{$y$} & \multirow[b]{2}{*}{$e_{y}$} & & \multicolumn{10}{|c|}{ pending rate $1 / \mathrm{w}$ : } \\
\hline & & & 0.0 & 0 & 0 & 0.04 & 0.05 & 0.06 & 0.07 & 0.08 & 0.09 & .10 \\
\hline 55 & 7.31 & 6 & 0 & $0.7 \%$ & $2.8 \%$ & & $\%$ & 2 & & $\%$ & & $56.5 \%$ \\
\hline 60 & 22.9 & 0 & 0 & 0.6 & & 0.6 & $\%$ & 1 & $\%$ & 3 & 4 & 48.7 \\
\hline 65 & 1 & 4 & 0. & $0.4 \%$ & 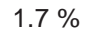 & 4. & $8.0 \%$ & $\%$ & $\%$ & $\%$ & . & 39. \\
\hline 70 & 1... & 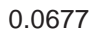 & 0.0 & ser & T. & 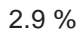 & on & a & $\%$ & $18.7 \%$ & 24 & 00.1 \\
\hline 10 & 11.12 & 0.0075 & 0.0 & & & & & & & $12.1 \%$ & & 20.3 \\
\hline 80 & 7.91 & 0.1264 & 0.0 & 0.1 & 0.4 & 1.0 & 1 & 3. & 4.7 & $6.7 \%$ & 9.0 & 11.6 \\
\hline 85 & 32 & 1880 & $0.0 \%$ & $0.0 \%$ & $0.2 \%$ & $0.4 \%$ & $0.8 \%$ & $1.4 \%$ & 2.110 & $3.0 \%$ & $4.1 \%$ & $5.5 \%$ \\
\hline
\end{tabular}

Tables $5-7$ regard the problem of unsustainable pensions in an opposite way which seems to be more practical: they contain (again separately for males and females in the Czech Republic in 2010) the maximal spending rates $1 / w$ that are admissible with a given 
tolerance (i.e. with a given probability of ruin which is yet tolerated); the calculations are performed for various values of investment drifts $\mu=0 \%, 0.5 \%, 1 \%, 1.5 \%, 2 \%, 2.5 \%$, $3 \%, 4 \%, \ldots, 7 \%$, only for single value of investment volatility $\sigma=5 \%$ and for various values of tolerance, namely $1 \%$ in Table 5, 5\% in Table 6 and $10 \%$ in Table 7 :

Table 5

\section{The Maximal Spending Rates with Tolerance of $1 \%$}

\begin{tabular}{|c|c|c|c|c|c|c|c|c|c|c|c|c|c|}
\hline \multicolumn{14}{|c|}{ Males CZ 2010 for $\sigma=5 \%$ : maximal spending rate $1 / \mathrm{w}(\%)$ with tolerance of $1 \%$} \\
\hline \multirow[b]{2}{*}{$x:$} & \multirow[b]{2}{*}{$e_{x}$} & \multirow[b]{2}{*}{$\lambda$} & \multicolumn{11}{|c|}{ Drift $\mu$ : } \\
\hline & & & $0 \%$ & $0.5 \%$ & $1 \%$ & $1.5 \%$ & $2 \%$ & $2.5 \%$ & $3 \%$ & $4 \%$ & $5 \%$ & $6 \%$ & $7 \%$ \\
\hline 55 & 22.50 & 0.0444 & $0.857 \%$ & $1.023 \%$ & $1.201 \%$ & $1.387 \%$ & $1.583 \%$ & $1.786 \%$ & $1.998 \%$ & $2.441 \%$ & $2.909 \%$ & $3.398 \%$ & $3.907 \%$ \\
\hline 60 & 18.69 & 0.0535 & $1.053 \%$ & $1.221 \%$ & $1.397 \%$ & $1.582 \%$ & $1.774 \%$ & $1.973 \%$ & $2.179 \%$ & $2.609 \%$ & $3.061 \%$ & $3.533 \%$ & $4.024 \%$ \\
\hline 65 & 15.26 & 0.0655 & $1.315 \%$ & $1.483 \%$ & $1.659 \%$ & $1.841 \%$ & $2.030 \%$ & $2.225 \%$ & $2.425 \%$ & $2.842 \%$ & $3.279 \%$ & $3.734 \%$ & $4.205 \%$ \\
\hline 70 & 12.11 & 0.0826 & $1.686 \%$ & $1.855 \%$ & $2.030 \%$ & $2.210 \%$ & $2.396 \%$ & $2.586 \%$ & $2.782 \%$ & $3.186 \%$ & $3.607 \%$ & $4.044 \%$ & $4.495 \%$ \\
\hline 75 & 9.16 & 0.1092 & $2.265 \%$ & $2.435 \%$ & $2.609 \%$ & $2.787 \%$ & $2.970 \%$ & $3.156 \%$ & $3.346 \%$ & $3.738 \%$ & $4.143 \%$ & $4.561 \%$ & $4.991 \%$ \\
\hline 80 & 6.62 & 0.1511 & $3.177 \%$ & $3.348 \%$ & $3.521 \%$ & $3.698 \%$ & $3.878 \%$ & $4.061 \%$ & $4.246 \%$ & $4.626 \%$ & $5.016 \%$ & $5.416 \%$ & $5.826 \%$ \\
\hline 85 & 4.54 & 0.2203 & $4.686 \%$ & $4.857 \%$ & $5.030 \%$ & $5.205 \%$ & $5.383 \%$ & $5.562 \%$ & $5.743 \%$ & $6.112 \%$ & $6.489 \%$ & $6.874 \%$ & $7.265 \%$ \\
\hline
\end{tabular}

\begin{tabular}{|c|c|c|c|c|c|c|c|c|c|c|c|c|c|}
\hline \multicolumn{14}{|c|}{ Females CZ 2010 for $\sigma=5 \%$ : maximal spending rate $1 / \mathrm{w}(\%)$ with tolerance of $1 \%$} \\
\hline \multirow[b]{2}{*}{$y$} & & \multirow[b]{2}{*}{$\lambda$} & \multicolumn{11}{|c|}{ Drift $\mu$ : } \\
\hline & & & $0 \%$ & $0.5 \%$ & $1 \%$ & $1.5 \%$ & $2 \%$ & $2.5 \%$ & $3 \%$ & $4 \%$ & $5 \%$ & $6 \%$ & $7 \%$ \\
\hline 55 & 27.31 & 0.0366 & $0.687 \%$ & $0.853 \%$ & $1.031 \%$ & $1.220 \%$ & $1.420 \%$ & $1.629 \%$ & $1.847 \%$ & $2.305 \%$ & $2.790 \%$ & $3.299 \%$ & $3.829 \%$ \\
\hline 60 & 22.91 & 0.0436 & $840 \%$ & $1.006 \%$ & $1.183 \%$ & $1.370 \%$ & $1.566 \%$ & $1.770 \%$ & $1.982 \%$ & $2.427 \%$ & $2.896 \%$ & $3.387 \%$ & 3.898 \\
\hline 65 & 18.71 & 0.0534 & $1.052 \%$ & $1.220 \%$ & $1.396 \%$ & $1.580 \%$ & $1.773 \%$ & $1.972 \%$ & $2.178 \%$ & $2.608 \%$ & $3.060 \%$ & $3.532 \%$ & $4.023 \%$ \\
\hline 70 & 14.77 & 0.0677 & $1.362 \%$ & $1.531 \%$ & $1.706 \%$ & $1.888 \%$ & $2.076 \%$ & $2.270 \%$ & $2.470 \%$ & $2.885 \%$ & $3.320 \%$ & $3.772 \%$ & 4.24 \\
\hline 75 & 11.12 & 0.0899 & $1.846 \%$ & $2.015 \%$ & $2.190 \%$ & $2.369 \%$ & $2.554 \%$ & $2.743 \%$ & $2.937 \%$ & $3.337 \%$ & $3.753 \%$ & $4.184 \%$ & $4.628 \%$ \\
\hline 80 & 7.91 & 0.1264 & $2.641 \%$ & $2.811 \%$ & $2.985 \%$ & $3.162 \%$ & $3.343 \%$ & $3.528 \%$ & $3.716 \%$ & $4.102 \%$ & $4.499 \%$ & $4.909 \%$ & $5.330 \%$ \\
\hline 85 & 5.32 & 0.1880 & $3.982 \%$ & $4.153 \%$ & $4.326 \%$ & $4.502 \%$ & $4.680 \%$ & $4.861 \%$ & $5.044 \%$ & $5.417 \%$ & $5.799 \%$ & $6.189 \%$ & 6.58 \\
\hline
\end{tabular}

Table 6

\section{The Maximal Spending Rates with Tolerance of $5 \%$}

\begin{tabular}{|c|c|c|c|c|c|c|c|c|c|c|c|c|c|}
\hline \multicolumn{14}{|c|}{ Males CZ 2010 for $\sigma=5 \%$ : maximal spending rate $1 / \mathrm{w}(\%)$ with tolerance of $5 \%$} \\
\hline \multirow[b]{2}{*}{$y$} & \multirow[b]{2}{*}{$e_{x}$} & & \multicolumn{11}{|c|}{ Drift $\mu$ : } \\
\hline & & & $0 \%$ & $0.5 \%$ & $1 \%$ & $1.5 \%$ & $2 \%$ & $2.5 \%$ & $3 \%$ & $4 \%$ & $5 \%$ & $6 \%$ & $7 \%$ \\
\hline 55 & 22.50 & 0.0444 & $2.284 \%$ & $1.919 \%$ & $2.180 \%$ & $2.449 \%$ & $2.725 \%$ & $3.007 \%$ & $3.296 \%$ & $3.890 \%$ & $4.503 \%$ & $5.133 \%$ & $5.778 \%$ \\
\hline 60 & 18.69 & 0.0535 & $2.783 \%$ & $2.290 \%$ & $2.550 \%$ & $2.816 \%$ & $3.090 \%$ & $3.368 \%$ & $3.653 \%$ & $4.236 \%$ & $4.837 \%$ & $5.454 \%$ & $6.085 \%$ \\
\hline 65 & 15.2 & 0.0655 & $3.445 \%$ & $2.781 \%$ & $3.041 \%$ & $3.306 \%$ & $3.576 \%$ & $3.852 \%$ & $4.132 \%$ & $4.705 \%$ & $5.294 \%$ & $5.897 \%$ & $6.513 \%$ \\
\hline 70 & 12.11 & 0.0826 & $4.384 \%$ & $3.478 \%$ & $3.737 \%$ & $4.000 \%$ & $4.268 \%$ & $4.540 \%$ & $4.816 \%$ & $5.379 \%$ & $5.955 \%$ & $6.544 \%$ & $7.144 \%$ \\
\hline 75 & 9.16 & 0.1092 & $5.849 \%$ & $4.566 \%$ & $4.824 \%$ & $5.086 \%$ & $5.351 \%$ & $5.619 \%$ & $5.891 \%$ & $6.443 \%$ & $7.007 \%$ & $7.580 \%$ & $8.164 \%$ \\
\hline 80 & 6.62 & 0.1511 & $8.156 \%$ & $6.278 \%$ & $6.536 \%$ & $6.796 \%$ & $7.059 \%$ & $7.324 \%$ & $7.592 \%$ & $8.134 \%$ & $8.686 \%$ & $9.245 \%$ & $9.813 \%$ \\
\hline 85 & 4.54 & 0.2203 & $11.969 \%$ & $9.108 \%$ & $9.365 \%$ & $9.624 \%$ & $9.885 \%$ & $10.148 \%$ & $10.412 \%$ & $10.946 \%$ & $11.486 \%$ & $12.032 \%$ & $12.585 \%$ \\
\hline
\end{tabular}


Table 6

\begin{tabular}{|c|c|c|c|c|c|c|c|c|c|c|c|c|c|}
\hline \multirow[b]{3}{*}{$x$} & \multicolumn{13}{|c|}{ Females CZ 2010 for $\sigma=5 \%$ : maximal spending rate $1 / \mathrm{w}(\%)$ with tolerance of $5 \%$} \\
\hline & \multirow[b]{2}{*}{$e_{y}$} & \multirow[b]{2}{*}{$\lambda$} & \multicolumn{11}{|c|}{ Drift $\mu$ : } \\
\hline & & & $0 \%$ & $0.5 \%$ & $1 \%$ & $1.5 \%$ & $2 \%$ & $2.5 \%$ & $3 \%$ & $4 \%$ & $5 \%$ & $6 \%$ & $7 \%$ \\
\hline 55 & 27.31 & 0.0366 & $1.348 \%$ & $1.599 \%$ & $1,861 \%$ & $2.132 \%$ & $2.411 \%$ & $2.698 \%$ & $2.992 \%$ & $3.598 \%$ & $4.225 \%$ & $4.870 \%$ & $5.530 \%$ \\
\hline 60 & 22.91 & 0.0436 & $1.635 \%$ & $1.887 \%$ & $2,147 \%$ & $2.416 \%$ & $2.693 \%$ & $2.976 \%$ & $3.265 \%$ & $3.860 \%$ & $4.474 \%$ & $5.106 \%$ & $5.752 \%$ \\
\hline 65 & 18.71 & 0.0534 & $2.035 \%$ & $2.287 \%$ & $2,547 \%$ & $2.814 \%$ & $3.087 \%$ & $3.366 \%$ & $3.651 \%$ & $4.234 \%$ & $4.835 \%$ & $5.452 \%$ & $6.083 \%$ \\
\hline 70 & 14.77 & 0.0677 & $2.617 \%$ & $2.870 \%$ & $3,130 \%$ & $3.394 \%$ & $3.664 \%$ & $3.939 \%$ & $4.219 \%$ & $4.790 \%$ & $5.377 \%$ & $5.978 \%$ & $6.592 \%$ \\
\hline 75 & 11.12 & 0.0899 & $3.525 \%$ & $3.779 \%$ & $4,037 \%$ & $4.300 \%$ & $4.567 \%$ & $4.838 \%$ & $5.112 \%$ & $5.672 \%$ & $6.244 \%$ & $6.828 \%$ & $7.423 \%$ \\
\hline 80 & 7.91 & 0.1264 & $5.016 \%$ & $5.271 \%$ & $5,529 \%$ & $5.790 \%$ & $6.054 \%$ & $6.321 \%$ & $6.591 \%$ & $7.138 \%$ & $7.696 \%$ & $8.263 \%$ & $8.839 \%$ \\
\hline 85 & 5.32 & 0.1880 & $7.532 \%$ & $7.787 \%$ & $8,045 \%$ & $8.304 \%$ & $8.566 \%$ & $8.830 \%$ & $9.095 \%$ & $9.632 \%$ & $10.177 \%$ & $10.728 \%$ & $11.286 \%$ \\
\hline
\end{tabular}

Table 7

\section{The Maximal Spending Rates with Tolerance of $10 \%$}

\begin{tabular}{|c|c|c|c|c|c|c|c|c|c|c|c|c|c|}
\hline \multicolumn{10}{|c|}{ Males CZ 2010 for $\boldsymbol{\sigma}=\mathbf{5} \%$ : maximal spending rate 1/w (\%) with tolerance of 10\% } \\
\hline \multirow{2}{*}{$\boldsymbol{x}$} & $\mathbf{e}_{\boldsymbol{x}}$ & $\boldsymbol{1}$ & $\mathbf{0} \%$ & $\mathbf{0 . 5} \%$ & $\mathbf{1} \%$ & $\mathbf{1 . 5} \%$ & $\mathbf{2} \%$ & $\mathbf{2 . 5} \%$ & $\mathbf{3} \%$ & $\mathbf{4} \%$ & $\mathbf{5} \%$ & $\mathbf{6} \%$ & $\mathbf{7} \%$ \\
\hline $\mathbf{5 5}$ & 22.50 & 0.0444 & $2.284 \%$ & $2.587 \%$ & $2.897 \%$ & $3.214 \%$ & $3.536 \%$ & $3.864 \%$ & $4.198 \%$ & $4.877 \%$ & $5.573 \%$ & $6.282 \%$ & $7.003 \%$ \\
\hline $\mathbf{6 0}$ & 18.69 & 0.0535 & $2.783 \%$ & $3.086 \%$ & $3.396 \%$ & $3.711 \%$ & $4.031 \%$ & $4.356 \%$ & $4.686 \%$ & $5.357 \%$ & $6.043 \%$ & $6.741 \%$ & $7.451 \%$ \\
\hline $\mathbf{6 5}$ & 15.26 & 0.0655 & $3.445 \%$ & $3.749 \%$ & $4.058 \%$ & $4.371 \%$ & $4.690 \%$ & $5.012 \%$ & $5.338 \%$ & $6.001 \%$ & $6.676 \%$ & $7.364 \%$ & $8.061 \%$ \\
\hline $\mathbf{7 0}$ & 12.11 & 0.0826 & $4.384 \%$ & $4.688 \%$ & $4.996 \%$ & $5.309 \%$ & $5.625 \%$ & $5.944 \%$ & $6.267 \%$ & $6.921 \%$ & $7.587 \%$ & $8.262 \%$ & $8.947 \%$ \\
\hline $\mathbf{7 5}$ & 9.16 & 0.1092 & $5.849 \%$ & $6.153 \%$ & $6.461 \%$ & $6.772 \%$ & $7.086 \%$ & $7.403 \%$ & $7.722 \%$ & $8.368 \%$ & $9.023 \%$ & $9.686 \%$ & $10.358 \%$ \\
\hline $\mathbf{8 0}$ & 6.62 & 0.1511 & $8.156 \%$ & $8.462 \%$ & $8.769 \%$ & $9.079 \%$ & $9.391 \%$ & $9.705 \%$ & $10.021 \%$ & $10.658 \%$ & $11.303 \%$ & $11.955 \%$ & $12.613 \%$ \\
\hline $\mathbf{8 5}$ & 4.54 & 0.2203 & $11.969 \%$ & $12.275 \%$ & $12.582 \%$ & $12.891 \%$ & $13.201 \%$ & $13.513 \%$ & $13.826 \%$ & $14.457 \%$ & $15.092 \%$ & $15.733 \%$ & $16.379 \%$ \\
\hline
\end{tabular}

\begin{tabular}{|c|c|c|c|c|c|c|c|c|c|c|c|c|c|}
\hline \multicolumn{14}{|c|}{ Females CZ 2010 for $\sigma=5 \%$ : maximal spending rate $1 / \mathrm{w}(\%)$ with tolerance of $10 \%$} \\
\hline \multirow[b]{2}{*}{$y$} & \multirow[b]{2}{*}{$\mathbf{e}_{y}$} & \multirow[b]{2}{*}{$\lambda$} & \multicolumn{11}{|c|}{ Drift $\mu$ : } \\
\hline & & & $0 \%$ & $0.5 \%$ & $1 \%$ & $1.5 \%$ & $2 \%$ & $2.5 \%$ & $3 \%$ & $4 \%$ & $5 \%$ & $6 \%$ & $7 \%$ \\
\hline 55 & 27.31 & 0.0366 & $1.854 \%$ & $2.155 \%$ & $2.466 \%$ & $2.785 \%$ & $3.110 \%$ & $3.442 \%$ & $3.779 \%$ & $4.469 \%$ & $5.175 \%$ & $5.896 \%$ & $6.630 \%$ \\
\hline 60 & 22.91 & 0.0436 & $2.240 \%$ & $2.543 \%$ & $2.853 \%$ & $3.170 \%$ & $3.493 \%$ & $3.821 \%$ & $4.155 \%$ & $4.836 \%$ & $5.532 \%$ & $6.242 \%$ & $6.965 \%$ \\
\hline 65 & 18.71 & 0.0534 & $2.780 \%$ & $3.083 \%$ & $3.392 \%$ & $3.708 \%$ & $4.028 \%$ & $4.353 \%$ & $4.683 \%$ & $5.354 \%$ & $6.040 \%$ & $6.738 \%$ & $7.448 \%$ \\
\hline 70 & 14.77 & 0.0677 & $3.565 \%$ & $3.869 \%$ & $4.177 \%$ & $4.491 \%$ & $4.809 \%$ & $5.131 \%$ & $5.456 \%$ & $6.118 \%$ & $6.792 \%$ & $7.477 \%$ & $8.173 \%$ \\
\hline 75 & 11.12 & 0.0899 & $4.789 \%$ & $5.093 \%$ & $5.401 \%$ & $5.713 \%$ & $6.029 \%$ & $6.347 \%$ & $6.669 \%$ & $7.320 \%$ & $7.982 \%$ & $8.654 \%$ & $9.335 \%$ \\
\hline 80 & 7.91 & 0.1264 & $6.799 \%$ & $7.104 \%$ & $7.412 \%$ & $7.722 \%$ & $8.035 \%$ & $8.350 \%$ & $8.668 \%$ & $9.310 \%$ & $9.960 \%$ & $10.618 \%$ & $11.283 \%$ \\
\hline 85 & 5.32 & 0.1880 & $10.190 \%$ & $10.496 \%$ & $10.803 \%$ & $11.112 \%$ & $11.423 \%$ & $11.736 \%$ & $12.050 \%$ & $12.683 \%$ & $13.322 \%$ & $13.967 \%$ & $14.618 \%$ \\
\hline
\end{tabular}

Finally Figures 3 and 4 describe the situation graphically: the growth of probabilities of unsustainable pension in dependence on growing spending rates (see Figure 3) and the decline of the minimal amount (i.e. the pension account) for unit life annuity in dependence on growing retirement age (see Figure 4): 


\section{Figure 3}

The Probabilities of Unsustainable Pension ( $\mu=2.5 \%$ and $\sigma=5 \%$ )

Males and females CZ 2010

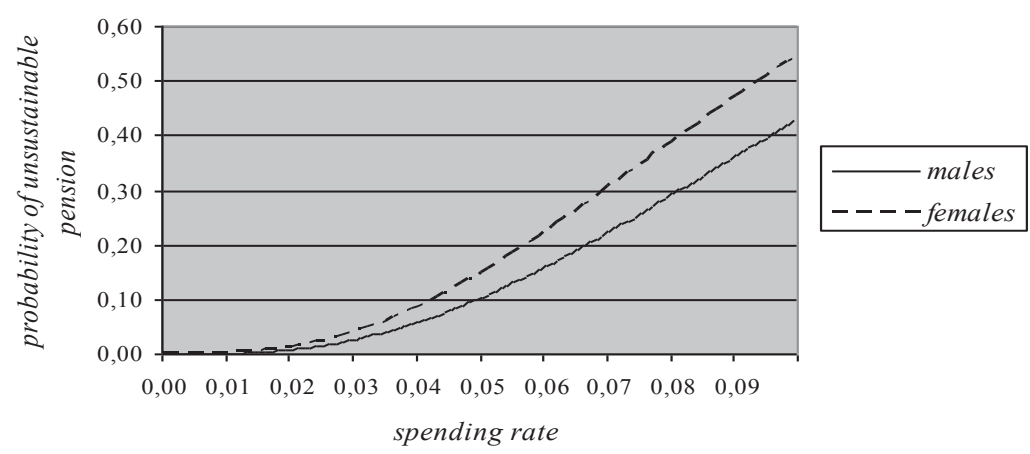

Figure 4

The Minimal Capital for Unit Life Annuity with Given Tolerance $(\mu=2.5 \%$ and $\sigma=5 \%)$

Males CZ 2010:

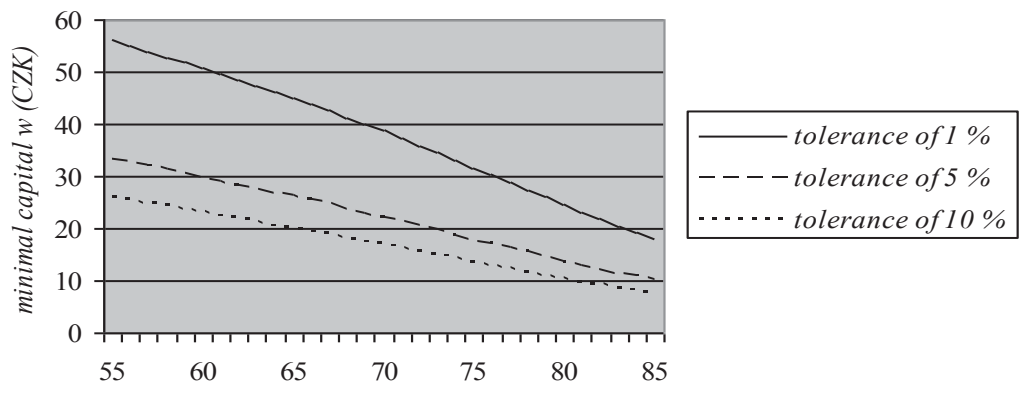

The previous results provide very interesting hints for future participants of pension plans, financial consultants, sales representatives of commercial insurance companies and managements of pension funds.

E.g. under a conservative investment strategy with $\mu=1 \%$ and $\sigma=5 \%$ a male with retirement age of 65 and spending rate 0.06 (i.e. CZK60,000 annually from a pension account of $\mathrm{CZK} 1,000,000)$ faces the unsustainable pension with probability $23.0 \%$ (naturally for a female of the same age this probability is higher, namely $32.1 \%$, see Table 2). In other words each fifth male and each third female are ruined before death according to this scenario. However, if the investment drift increases than (even for higher investment volatility) the ruin probability decreases significantly, e.g. for $\mu=5 \%$ and $\sigma=10 \%$ the ruin probabilities are only $9.7 \%$ for males and $13.0 \%$ for females (see Table 4), i.e. only each tenth male and each eighth female are ruined. 
Similarly, under the tolerance of $1 \%$, retirement age 65 and conservative investment strategy with $\mu=1 \%$ and $\sigma=5 \%$ a male may spend from his pension account of CZK 1000,000 not more than CZK 16,590 annually (i.e. CZK 1,383 monthly) and a female of the same age not more than CZK13,960 (i.e. CZK1,163 monthly, see Table 5). These values are surprisingly low but the reason is very simple, namely the high level of safety with tolerance of only $1 \%$. For higher investment drift $\mu=2.5 \%$ ceteris paribus the maximal annual withdrawals are CZK22,250 for males and CZK19,720 for females. If the tolerance of $10 \%$ is sufficient (see Table 7) then under conservative investment parameters $\mu=1 \%$ and $\sigma=5 \%$ the male can draw CZK 40,580 annually (i.e. CZK 3,382 monthly) and the female CZK33,920 annually (i.e. CZK2,827 monthly). For higher investment drift $\mu=2.5 \%$ (which corresponds to the situation in the Czech Republic) ceteris paribus the male can spend CZK 50,120 annually (i.e. CZK4,177 monthly) and the female CZK43,530 annually (i.e. CZK3,628 monthly).

\section{Generation Life Tables for Pension System}

The Life Tables applied for the pension system and pension funds in the Czech Republic should respect two facts that are typical just for pensions: (1) decreasing trend of the rates of mortality in time and (2) selection of pension portfolios involving persons in better health conditions than the population standard. A possible solution is to construct the Generation (or Cohort) Life Tables (GLT) with projections distinguishing among particular generations (cohorts), see Cipra, 1998. Moreover, these Tables are adjusted in a special way to be suitable for pensions.

In the Period (or Current) Life Tables the probability of death $q_{x}$ (and the probability of survival) depends only on the age $x$ that is looked upon as the current age in the year for which the LT were constructed. Therefore e.g. $q_{65}$ from the LT for the Czech Republic in 2010 concerns the male generation 1945 . However, one uses this value approximately as the probability of death in the age $x$ also for other male cohorts. Better solutions consist in applying the GLT where the probability of death $q_{x}{ }^{\tau}$ depends also on the year of birth (therefore the probability discussed above should be denoted as $q_{65}{ }^{1945}$ ). In addition, this principle must be respected also for multiyear probabilities, e.g.

$$
{ }_{5} p_{65}^{1945}=p_{65}^{1945} \cdot p_{66}^{1945} \cdot p_{67}^{1945} \cdot p_{68}^{1945} \cdot p_{69}^{1945}
$$

The application of the GLT can be recommended just for the Czech Republic with a high longevity risk in the pension systems.

Figures $5 \mathrm{a}-5 \mathrm{~b}$ show numbers of survivors $l_{x}$ and $l_{y}$ for male and female generations 1935 , 1955 a 1975 according to the GLT for the Czech Republic constructed by Cipra, 1998. These GLT respect another important fact, namely the selectivity of annuity portfolios (it is obvious since the life annuities are preferred by persons without health problems, see also Schmithals, B., Schütz, EU, 1995. 
Figure $5 a$

Numbers of Survivors $I_{x}$ for Male Generations 1935, 1955 a 1975 according to the GLT for the Czech Republic

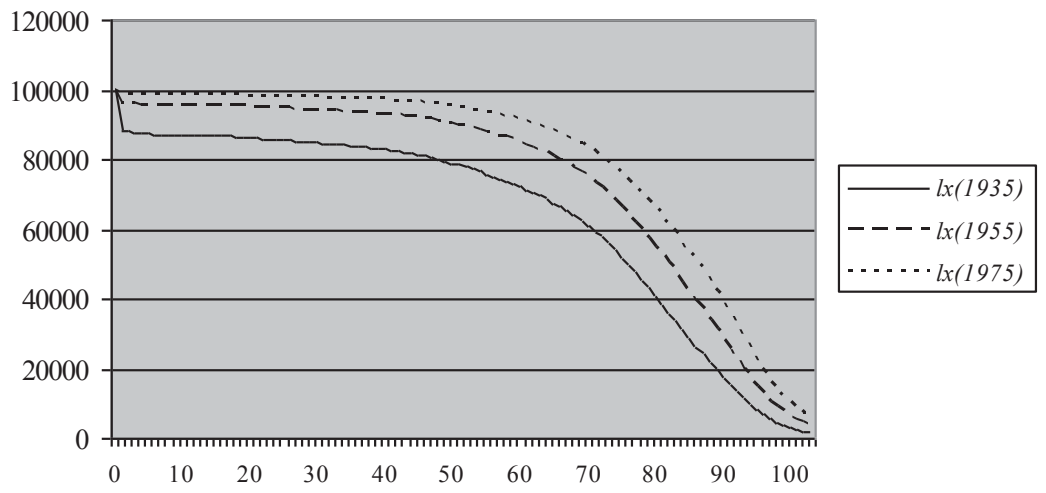

Source: Cipra, 1998.

Figure $5 b$

Numbers of Survivors $I_{y}$ for Female Generations 1935, 1955 a 1975 according to the GLT for the Czech Republic

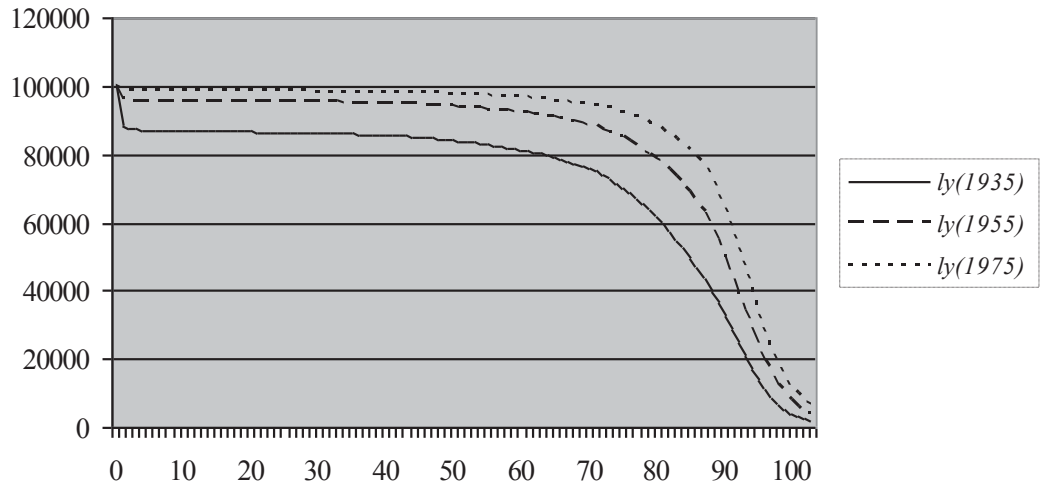

Source: Cipra, 1998.

When applying the GLT the differences are not negligible from the financial point of view if one compares it with the application of the Period LT. Table 8 contains the monthly payments for life annuities paid off from the age 60 achieved in various years if the pension account in the age 60 is CZK1,000,000. The three alternative calculation formulas are $1,000,000 / 12 \cdot e_{60}$ (where $e_{60}$ is the life expectancy in the age 60) or $1,000,000 / 12 \ddot{a}_{60}(4 \%)$ (where $\ddot{a}_{60}(4 \%)$ is the annuity factor in the age 60 for the interest rate $4 \%$ p.a.) or $1,000,000 / 12 \cdot \ddot{a}_{60}(6 \%)$ (where $\ddot{a}_{60}(6 \%)$ is the annuity factor in the age 60 for the interest rate $6 \%$ p.a.). E.g. for the interest rate $4 \%$ p.a. and for the male generation 
2010 in the age 60 the monthly annuity payment is CZK5,581. On the other hand, if applying the classical Period LT the monthly annuity payment would be substantially higher, namely CZK 7,111, since the Period LT do not reflect the increasing longevity.

Table 8

Annual Payment for Life Annuity Using Generation and Current LF

\begin{tabular}{|l|c|c|c|c|c|c|}
\hline \multirow{2}{*}{ Monthly payment (in CZK) for life annuity (the pension account of CZK1,000,000 in age 60) } \\
\hline \multirow{2}{*}{$\begin{array}{l}\text { Pension } \\
\text { from year: }\end{array}$} & \multicolumn{2}{|c|}{ Male in age $\mathbf{6 0}$ by means of: } & \multicolumn{3}{c|}{ Female in age $\mathbf{6 0}$ by means of: } \\
\cline { 2 - 7 } & $\mathbf{e}_{60}=\ddot{a}_{60}(\mathbf{0} \%)$ & $\ddot{a}_{60}(\mathbf{4} \%)$ & $\ddot{a}_{60}(\mathbf{6} \%)$ & $\mathbf{e}_{60} \mathbf{a}_{60} \mathbf{( 0 \% )}$ & $\ddot{a}_{60}(\mathbf{4} \%)$ & $\ddot{a}_{60}(\mathbf{6} \%)$ \\
\hline $\mathbf{1 9 9 6}$ & 3810 & 5859 & 7011 & 3153 & 5119 & 6250 \\
\hline $\mathbf{2 0 0 0}$ & 3727 & 5775 & 6928 & 3076 & 5039 & 6171 \\
\hline $\mathbf{2 0 0 5}$ & 3626 & 5675 & 6829 & 2990 & 4948 & 6081 \\
\hline $\mathbf{2 0 1 0}$ & 3534 & 5581 & 6737 & 2913 & 4867 & 6001 \\
\hline $\mathbf{2 0 1 5}$ & 3446 & 5492 & 6649 & 2844 & 4795 & 5930 \\
\hline $\mathbf{2 0 2 0}$ & 3364 & 5408 & 6566 & 2782 & 4730 & 5867 \\
\hline $\mathbf{2 0 2 5}$ & 3287 & 5329 & 6488 & 2728 & 4673 & 5810 \\
\hline $\mathbf{2 0 3 0}$ & 3215 & 5254 & 6414 & 2679 & 4621 & 5759 \\
\hline Current LT & 5128 & 7111 & 8235 & 4087 & 6010 & 7106 \\
\hline
\end{tabular}

Source: Cipra, 1998.

\section{Unisex Life Tables for Pension System}

This section can be looked upon as a reaction to the movement against the discrimination using the gender as a calculation parameter in the life insurance and pension systems. The Unisex Life Tables (ULT) presented here (see also Smetana and Cipra, 2005) have been constructed applying the same methodology as the Czech Statistical Office for the gender depending LT (see more in Cipra, 1998). The comparison of the net regular annual premiums (i.e. the annual net price paid during the deferment period) for the deferred life annuities is added.

Figure 6 (the probabilities of death $q_{x}$ according to the ULT for the Czech Republic in 2003) confirms the expectation that the unisex mortality is approximately an average of the male and female mortality. One should note that the population data used in the paper refer to various years (e.g. 2003, 2008 and 2010) but annuity products within particular decades use to be comparable if one compares prices. 


\section{Figure 6}

Probabilities of Death $q_{x}$ according to the ULT for the Czech Republic in 2003

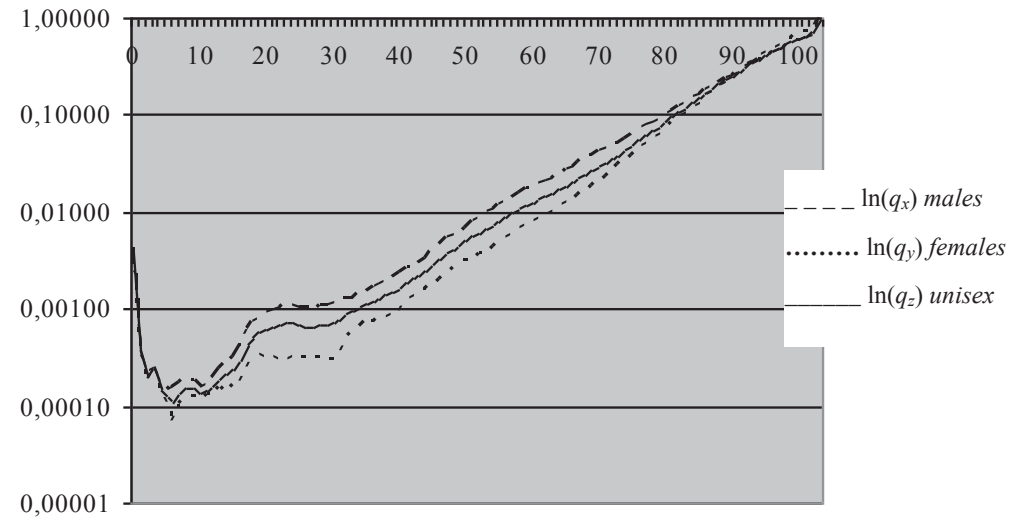

Source: Smetana and Cipra, 2005.

Tables $9 \mathrm{a}-9 \mathrm{~b}$ present single and regular annual premium for deferred life annuities with annual payments of CZK12,000 for the Czech Republic in 2003 (the technical interest rate is $2.4 \%$ p.a.). The tables demonstrate the fact that the analysis of longevity risk must be modified if respecting the gender effect.

Table 9a

Single Premium for Deferred Life Annuities with Annual Payments of CZK12,000 for the Czech Republic in 2003 (2.4\% p.a.)

\begin{tabular}{|l|l|r|r|r|r|r|c|}
\hline Age & $\begin{array}{l}\text { Defer- } \\
\text { ment }\end{array}$ & Unisex (U) & Males $(\boldsymbol{M})$ & Females $(\boldsymbol{F})$ & $\begin{array}{c}\text { Ratio U/M } \\
\mathbf{( \% )}\end{array}$ & $\begin{array}{c}\text { Ratio U/F } \\
(\mathbf{\%})\end{array}$ & $\begin{array}{c}\text { Ratio } \boldsymbol{M} / \boldsymbol{F} \\
\mathbf{( \% )}\end{array}$ \\
\hline $\mathbf{2 0}$ & $\mathbf{4 0}$ & 58629 & 50595 & 71864 & $115,88 \%$ & $81,58 \%$ & $70,40 \%$ \\
\hline $\mathbf{3 0}$ & $\mathbf{3 0}$ & 74821 & 64791 & 91382 & $115,48 \%$ & $81,88 \%$ & $70,90 \%$ \\
\hline $\mathbf{4 0}$ & $\mathbf{2 0}$ & 95880 & 83397 & 116564 & $114,97 \%$ & $82,26 \%$ & $71,55 \%$ \\
\hline $\mathbf{5 0}$ & $\mathbf{1 0}$ & 125114 & 110017 & 150445 & $113,72 \%$ & $83,16 \%$ & $73,13 \%$ \\
\hline
\end{tabular}

Source: Smetana and Cipra, 2005.

Table 9b

Regular Annual Premium for Deferred Life Annuities with Annual Payments of CZK12,000 for the Czech Republic in 2003 (2.4 \% p.a.)

\begin{tabular}{|l|l|c|c|c|c|c|c|}
\hline Age & $\begin{array}{l}\text { Defer- } \\
\text { ment }\end{array}$ & Unisex $(\boldsymbol{U})$ & Males $(\boldsymbol{M})$ & Females $(\boldsymbol{F})$ & $\begin{array}{c}\text { Ratio U/M } \\
(\mathbf{\%})\end{array}$ & $\begin{array}{c}\text { Ratio U/F } \\
\mathbf{( \% )}\end{array}$ & $\begin{array}{c}\text { Ratio } \boldsymbol{M} / \boldsymbol{F} \\
(\mathbf{\%})\end{array}$ \\
\hline $\mathbf{2 0}$ & $\mathbf{4 0}$ & 2293 & 1997 & 2784 & $114,81 \%$ & $82,36 \%$ & $71,74 \%$ \\
\hline $\mathbf{3 0}$ & $\mathbf{3 0}$ & 3534 & 3091 & 4272 & $114,33 \%$ & $82,72 \%$ & $72,35 \%$ \\
\hline $\mathbf{4 0}$ & $\mathbf{2 0}$ & 6134 & 5398 & 7370 & $113,62 \%$ & $83,23 \%$ & $73,25 \%$ \\
\hline $\mathbf{5 0}$ & $\mathbf{1 0}$ & 14287 & 12712 & 16986 & $112,40 \%$ & $84,12 \%$ & $74,84 \%$ \\
\hline
\end{tabular}

Source: Smetana and Cipra, 2005. 


\section{Conclusions}

The paper has concentrated on three important aspects that must be taken into account when constructing products burdened by longevity risk: (1) the sustainability of pensions, (2) the generation longevity and (3) the gender longevity. It has been shown numerically that these aspects really play a very important role for the pensions in the Czech Republic.

In order to obtain the results presented in the paper it has been necessary to derive a corresponding mathematical background (see e.g. Section 2 for the sustainability of pensions) and then to calibrate it using Czech demographic and financial data. The analysis of generation longevity and gender longevity has been performed abroad (e.g. in Germany and UK) but the results for the Czech Republic presented in the paper have been (in the time of their origin) the first trials with the Czech data.

The models in this contribution enable to investigate the probability of unsustainability of pensions respecting the randomness of investment activities and longevity. They give numerical confirmation that such a probability decreases with increasing retirement age, with decreasing spending ratio (in particular with increasing pension account and decreasing annuity payments), with increasing investment drift, with decreasing investment volatility and that is always lower for males than for females. In particular for the Czech Republic, the analysis of such a type is up-to-date in the context of the pension reform which seems to be unavoidable nowadays (see also Cipra, 2012). The methods presented in the paper are examples of calculations which may be very useful (and some of them even necessary) in this context.

\section{References}

Blake, D., Orszag, J. M. (1999), "Annual Estimates of Personal Wealth Holdings in the United Kingdom since 1948." Applied Financial Economics, Vol. 9, pp. 397-421.

Cannon, E., Tonks, I. (2008), Annuity Market. Oxford: Oxford University Press.

Cipra, T. (1998), "Cohort Life Tables for Pension Insurance and Pension Funds in Czech Republic." Pojistné rozpravy, Vol. 3, pp. 31-57 (in Czech).

Cipra, T. (2008), Financial Econometrics. Praha: Ekopress (in Czech).

Cipra, T. (2010), Financial and Insurance Formulas. Heidelberg, Dordrecht, London, New York: Physica-Verlag / Springer.

Cipra, T. (2012), Pension: Quantitative Approach. Praha: Ekopress (in Czech).

Clark, G. L., Munnell A. H., Orszag, J. M., Eds. (2006), Pensions and Retirement Income. Oxford: Oxford University Press.

Dufresne, D. (1990), "The Distribution of a Perpetuity with Applications to Risk Theory and Pension Funding." Scandinavian Actuarial Journal, Vol. 9, pp. 39-79.

Malliaris, A. G., Brock, W. A. (1982), Stochastic Methods in Economics and Finance. Amsterdam: North-Holland.

Milevsky, M. A. (1997), "The Present Value of a Stochastic Perpetuity and the Gamma Distribution." Insurance: Mathematics and Economics, Vol. 20, pp. 243-250. 
Milevsky, M. A. (2006), The Calculus of Retirement Income. Cambridge: Cambridge University Press.

OECD (2011), Pensions at a Glance 2011: Retirement-income Systems in OECD and G20 Countries. Paris: OECD Publishing.

Schmithals, B., Schütz, E.U. (1995), "Herleitung der DAV-Sterbetafel 1994 R für Rentenversicherung." Blätter der DGVM, Vol. XXII, pp. 29-69.

Sheshinski, E. (2005), The Economic Theory of Annuities. Princeton: Princeton University Press.

Smetana, P., Cipra, T. (2005), "Unisex Life-Tables for the Czech Republic and Consequences for Insurance Calculations”. Pojistný obzor, Vol. 82, pp. 10-17 (in Czech).

Uebelmesser, S. (2004), Unfunded Pension Systems: Ageing and Migration. Amsterdam: Elsevier. 